\title{
Charge-State Reduction with Improved Signal Intensity of Oligonucleotides in Electrospray Ionization Mass Spectrometry
}

\author{
David C. Muddiman, Xueheng Cheng, ${ }^{*}$ Harold R. Udseth, and \\ Richard D. Smith \\ Macromolecular Structure and Dynamics, Pacific Northwest National Laboratory, \\ Environmental Molecular Sciences Laboratory, Richland, Washington, USA
}

\begin{abstract}
The shift of charge states of oligonucleotide negative ions formed in electrospray ionization mass spectrometry to higher mass-to-charge ratio has been accomplished by addition of organic acids and bases to the solution to be electrosprayed. The use of acetic acid or formic acid combined with piperidine and imidazole effectively reduced charge states. Signal intensity and stability were enhanced greatly when the infused solution contained a high percentage of acetonitrile. In addition, the cocktail that contained imidazole, piperidine, and acetic acid in $80 \%$ acetonitrile not only reduced charge states, but also substantially suppressed $\mathrm{Na}$ adduction. Several oligonucleotides that varied in base composition and length were investigated, and studies of mixtures showed a significant reduction in spectral complexity. (J Am Soc Mass Spectrom 1996, 7, 697-706)
\end{abstract}

$\mathrm{T}$ The ability to produce intact molecular ions of high molecular weight biopolymers is attributed largely to the advent of electrospray ionization (ESI) $[1-4]$ and matrix-assisted laser desorption ionization (MALDI) [5-8]. Due to the multiply charged ions produced in the ESI source, conventional mass analyzers can be employed effectively to analyze high molecular weight compounds. In general, the number of charges depends on the chemical nature and structure of the biopolymer (e.g., protein, ss-DNA) as well as the solution and gas-phase environments; however, large molecules typically exhibit a broad charge-state distribution centered at relatively low mass-to-charge ratios $(m / z<2000)$. Although the phenomena of multiple charging allows use of mass-to-charge limited mass analyzers (e.g., quadrupole), there is a substantial increase in the spectral complexity that prohibits direct analysis of complex mixtures (i.e., without a front-end separation step). The resulting complex mass spectrum is attributed primarily to the convolution of the charge-state distribution from each component in the mixture, which makes interpretation increasingly difficult due to insufficient mass resolution and reduced signal intensity for each peak. As a result, only a limited number of studies have been conducted on

Address reprint requests to Dr. Richard D. Smith, Environmental Molecular Sciences Laboratory, Pacific Northwest National Laboratory, Mail Stop P8-19, P.O. Box 999, Richland, WA 99352.

* Present address: Pharmaceutical Discovery Division, Abbott Laboratories, Abbott Park, IL 60064. complex mixtures by using electrospray ionizationmass spectrometry (ESI-MS) [9-11].

One of the most challenging and potentially significant applications of the use of ESI to analyze complex mixtures is in the area of DNA analyses; specifically, the analysis of chain-termination oligonucleotides produced by using Sanger methodology [12]. However, there are three major problems associated with the analysis of such DNA mixtures: (1) cation adduction to the polyanionic backbone, (2) spectral complexity due to multiple charge-state peaks of each component, and (3) obtaining detection limits comparable to electrophoretic methods. Each of these problems associated with mass spectrometric analysis of oligonucleotides has been addressed individually. The removal of ubiquitous cations (e.g., $\mathrm{Na}^{+}$and $\mathrm{K}^{+}$) to facilitate the precise mass measurement of nucleic acids has been studied by several researchers. Stults and Marsters [13] reported that simple precipitation of the oligomers out of solution with ammonium acetate produced a substantial improvement in spectrum quality. This improvement was attributed to a neutral loss of ammonia in the gas phase and enabled the analysis of a mixed base ss-DNA 77-mer with only one remaining sodium adduct.

A recent report by Greig and Griffey [14] indicated that organic bases effectively can reduce alkali adduction to ss-DNA. Several reports also have suggested the use of triethylamine to moderate $\mathrm{Na}$ and $\mathrm{K}$ adduction [15-18]. Greig and Griffey [14] also investigated 
other strong and weak organic bases; however, the use of piperidine resulted in a substantial decrease in $\mathrm{Na}$ adduction, but this was accompanied by a signal suppression of the analyte material. The addition of a weak base, imidazole, slightly reduced cation adduction with improved signal intensity. Interestingly, they also noted a shift to higher mass-to-charge ratio values (i.e., a reduction in the maximum charge state) upon addition of imidazole. Furthermore, the addition of $\mathrm{N}$-methyl imidazole had no effect on the ESI mass spectra; this led them to conclude that imidazole interacts with the polyanionic backbone with loss of neutral imidazole that occurs as the ion enters into the gas phase. The co-addition of piperidine and imidazole resulted in the enhancement of signal intensity, almost complete suppression of alkali adduct formation, and a slight shift of the ion charge states to higher mass-tocharge ratio [14].

Bleicher and Bayer [18] reported on the use of organic solvents to improve the ESI mass spectra of oligonucleotides. They concluded that the higher the percentage of organic phase, the higher the ion abundance. A sixfold increase in ion intensity was observed when the solution being infused contained $80 \%$ acetonitrile. It also was noted that addition of triethylamine suppressed the formation of salts.

Although the suppression of adduction and improved signal intensity have been demonstrated individually, it is uncertain if these properties can be maintained in the presence of a charge-state reduction agent (i.e., use of a single sample preparation technique). Cheng et al. [11] investigated different approaches to reduce the charge state of oligonucleotide ions; the approaches included addition of inorganic and organic acids to the DNA solution and formation of diamine adducts in solution followed by dissociation in the interface region. In principle, the reduction of charge states can be accomplished at several stages in the ESI process: solution conditions, in the ESI interface region, and in the mass spectrometer by using gas-phase reactions. These approaches are all feasible; however, the potential utilization of ESI-MS to sequence DNA rapidly requires a sample preparation method that does not introduce new "bottlenecks" with respect to the speed of analysis. Thus, although several approaches are possible, alteration of the solution composition to suppress cation adduction while reducing charge states would be the most attractive approach for two main reasons: (1) rapid sample preparation (automation) and (2) controlling the solution-phase chemistry is probably the most analytically reproducible.

The purpose of these investigations is to expand upon the original work on charge-state reduction [11] of ss-DNA by using solution-phase chemistry with the aim to improve the mass spectral quality (i.e., reduction of cation adduction and improved ion abundance). Our goal is to facilitate the use of ESI mass spectrometry to analyze complex mixtures of ss-DNA. We have evaluated our approach by using a six-component mixture.

\section{Experimental}

\section{Materials and Reagents}

Oligonucleotides $\left[\mathrm{d}(\mathrm{pC})_{12}, \mathrm{~d}(\mathrm{pT})_{18}, \mathrm{~d}(\mathrm{pA})_{8}, \mathrm{~d}(\mathrm{pC})_{8}\right.$, $d(p T)_{8}$, and $\left.d(p A)_{6}\right]$ were purchased from Sigma (St. Louis, MO) as sodium or ammonium salts. Desalting of $d(p T)_{18}$ and $d(p C)_{12}$ was accomplished via exchange with ammonium ions by using $100-\mathrm{mM}$ ammonium acetate and subsequent concentration by using Centricon-3 filters (Amicon, Beverly, MA). The process was repeated twice and the oligonucleotides were stored in 5-mM ammonium acetate until use. The smaller oligomers $(<10$-mer) were used as received. All other reagents were obtained at the highest purity available and were used without further purification.

\section{Mass Spectrometer}

A Finnigan (San Jose, CA) TSQ-7000 triple quadrupole mass spectrometer was utilized for this research. A coaxial $\mathrm{SF}_{6}$ sheath gas was used to suppress corona discharge. A sheathless ESI source optimized for the analysis of small volumes of aqueous solutions at low infusion rates $(0.2 \mu \mathrm{L} / \mathrm{min})$ was used [19]. A source potential of $2.2 \mathrm{kV}$ was applied to produce a stable negative ESI current. The capillary temperature was held constant at $160^{\circ} \mathrm{C}$ for all experiments unless otherwise noted.

\section{Sample Preparation}

Oligonucleotide solutions were prepared in the appropriate solvent(s) as indicated in the text with concentrations that ranged from 10 to $90 \mathrm{pmol} / \mu \mathrm{L}$. Each sample was infused directly into the ESI source by using a syringe pump (Harvard, South Natick, MA). All oligonucleotides were mixed with the appropriate amount of each reagent immediately before infusion to prevent possible decomposition of oligonucleotides. The final solution concentration of each reagent was as follows: acetic acid, $2.5 \mathrm{M}$; formic acid, $2.5 \mathrm{M}$; piperidine, $25 \mathrm{mM}$; imidazole, $25 \mathrm{mM}$; acetonitrile, 80 vol \%; sodium chloride, $10 \mathrm{mM}$.

\section{Results}

\section{Effect of Solution Composition on Electrospray Spectra of $T_{18}$}

The initial investigations of the solution conditions focused on the sodium salt of $d(\mathrm{pT})_{18}$. The sample was desalted by cation exchange with ammonium ions as described in the Experimental section. Figure la shows the ESI mass spectrum of $T_{18}$ obtained from a solution that contained $80 \%$ acetonitrile (ACN) that indicates a narrow charge-state distribution with the maximum 
charge state at $4^{-}$. A small amount of residual sodium adduction is observed; however, precise determination of molecular weight was obtained readily. The addition of $A C N$ produced a more intense analyte signal and stable ESI current as previously reported [18]. The ESI mass spectrum shown in Figure 1a serves as a reference spectrum for other solution conditions because $A C N$ is used throughout (unless otherwise noted).

Evaluation of several different solution conditions was conducted to determine if the analyte signal could be maintained while the charge states were simultaneously reduced. Figure $1 \mathrm{~b}$ shows the effect of the addition of 25-mM imidazole and 25-mM piperidine on the ESI mass spectrum of $T_{18}$. Inspection of Figure $1 b$ clearly indicates two major changes: (1) the extent of $\mathrm{Na}$ adduction has been reduced and (2) the maximum charge state has shifted from $4^{-}$to $3^{-}$(i.e., chargestate reduction). Addition of imidazole or piperidine alone reduced the charge state to the same extent (data not shown); however, piperidine was found to be more effective in suppression of cation adduction. It also should be mentioned that when only piperidine was added, cationization with $\mathrm{Na}$ was reduced substantially albeit with a suppression of analyte signal, consistent with previous reports [14], and this suggests that the addition of the weak base enhances spectral intensity. However, piperidine and imidazole are not

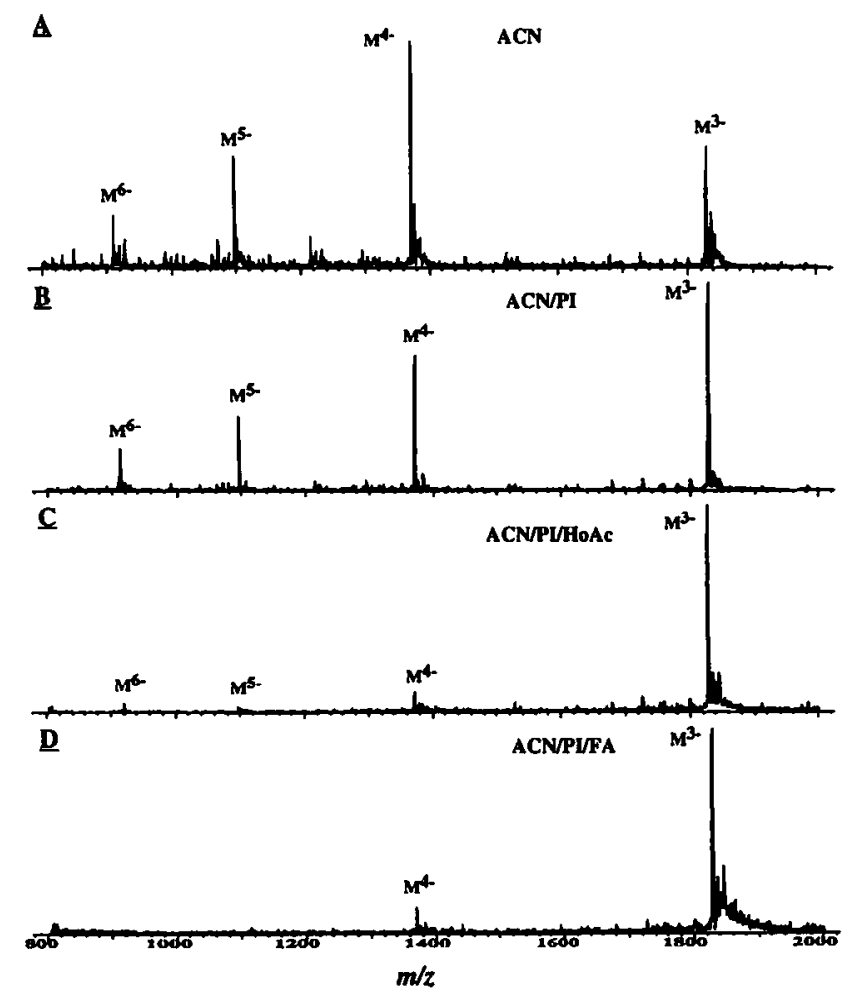

Figure 1. Electrospray ionization mass spectra of $d(\mathrm{pT})_{18}$ in (a) $80 \% \mathrm{ACN}$, (b) $80 \% \mathrm{ACN} / 25-\mathrm{mM}$ piperidine/25-mM imidazole, (c) $80 \% \mathrm{ACN} / 25-\mathrm{mM}$ piperidine $/ 25-\mathrm{mM}$ imidazole $/ 2.5-\mathrm{M}$ acetic acid, and (d) $80 \% \mathrm{ACN} / 25-\mathrm{mM}$ piperidine $/ 25-\mathrm{mM}$ imidazole/2.5-M formic acid. homologous; thus, the signal enhancing effect may or may not be related to the low basicity of imidazole.

Although the maximum charge state was shifted to higher mass-to-charge ratio by co-addition of imidazole and piperidine, a further reduction in the number of charge states (as well as the extent of charging) is also important for mixture analysis. To accomplish this end, two organic acids previously reported to reduce charge states most effectively [11] were investigated, namely, acetic acid (HOAc, $\mathrm{pK}_{a}=4.75$ ) and formic acid (FA, $\left.\mathrm{P} K_{a}=3.75\right)$. Figure $1 \mathrm{c}$ shows the ESI mass spectrum of $T_{18}$ obtained from a solution that contained piperidine $(25 \mathrm{mM})$, imidazole $(25 \mathrm{mM})$, and HOAc $(2.5 \mathrm{M})$ in $80 \% \mathrm{ACN}$. Note that almost all of the intensity is shifted to a single charge state $\left(3^{-}\right)$in the mass range investigated. Figure $1 \mathrm{~d}$ is the ESI mass spectrum of the same solution as Figure 1c except the HOAc is replaced with the stronger acid (FA, 2.5 M). Almost all the intensity once again is shifted into the $3^{-}$charge state; however, the $5^{-}$charge state is not observable and the $4^{-}$charge state is very weak when formic acid is employed as the charge-state reducing agent. This result is attributed to the lower $\mathrm{p} K_{a}$ of FA relative to HOAc.

The results of these solution conditions (Figure $1 a-d)$ and several others are illustrated in Figure 2a.

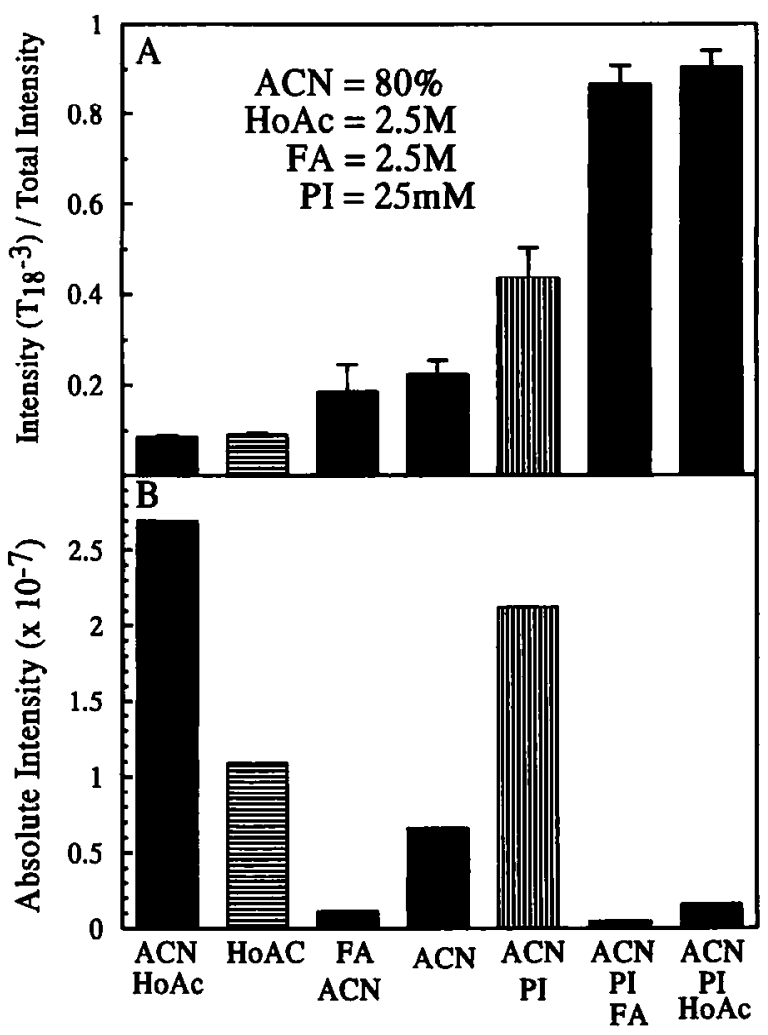

Figure 2. (a) Intensity of the $3^{-}$charge state of $d(\mathrm{pT})_{18}$ normalized to the total intensity of all observed charge states as a function of different solution compositions (HOAc, acetic acid; PI, piperidine and imidazole; $\mathrm{FA}$, formic acid; $\mathrm{ACN}$, acetonitrile). Error bars represent the confidence interval of the mean (95\% confidence limits) of six determinations. (b) Absolute intensity of $\mathrm{d}(\mathrm{pT})_{18}$ as a function of solution composition. 
The extent of charge-state reduction (abscissa) is expressed as the intensity of the $T_{18}^{3-}$ relative to the total intensity of all observed charge states. The error is expressed as the confidence interval of the mean $\mu=$ $x \pm t s / \sqrt{ } N$, where $\mu$ is the true value, $x$ is the experimental mean, $t$ is the $t$ statistic at the $95 \%$ confidence level, and $N=6$ determinations. Several interesting observations should be noted from the results presented in Figure 2a. First, the addition of HOAc alone and in the presence of ACN did not result in significant charge-state shifting, whereas co-addition of piperidine and imidazole to a solution that contained HOAc almost completely shifted all the intensity into the $3^{-}$charge state. This result was also true for a solution that contained only FA and ACN; however, a solution that contained FA in deionized water appeared to reduce charge states effectively but with very poor intensity (data not shown). The poor intensity is attributed to the competition of FA with the oligonucleotide in the ionization process. A sample preparation method to reduce charge states will be valuable only if the charge-state reduction is not associated with a suppression of absolute signal intensity. Figure $2 \mathrm{~b}$ shows the absolute signal intensity (sum of all observed charge states) of the different mixtures used to improve spectral quality while reducing charge states. Thus, although FA in $80 \%$ ACN slightly shifted the charge states to higher mass-to-charge ratio, a substantial reduction in ion yield also was observed. The co-addition of piperidine and imidazole along with the formic acid or acetic acid shifted the charge state almost completely to the higher observable charge state $\left(3^{-}\right)$in the mass-to-charge ratio range investigated. Oligonucleotides infused from a solution that contained only $80 \% \mathrm{ACN}$ appeared to slightly reduce charge states when compared to spraying them directly from an aqueous solution. The loss in absolute signal intensity when HOAc and FA are added in the presence of piperidine and imidazole will be addressed vide infra.

Although the $d\left(\mathrm{pT}_{18}\right)$ used in the foregoing experiment was desalted, it was still uncertain how much the addition of piperidine could effectively suppress the sodium contamination. In an attempt to "quantify" how much Na could be removed, a known amount of $\mathrm{NaCl}$ was added to the solution that contained the oligonucleotide. Figure 3a shows a spectrum of a desalted $T_{18}(20 \mu \mathrm{M})$ obtained from a solution that contained $80-\mathrm{vol} \% \mathrm{ACN}$ that contained $10-\mathrm{mM} \mathrm{NaCl}$ (a dynamic equilibrium was established prior to analysis). The intensity is very poor and severe cationization with $\mathrm{Na}$ is observed; the maximum for the $4^{-}$charge state was $[\mathrm{M}+4 \mathrm{Na}]^{4^{-}}$and that for the $6^{-}$charge state was $\left[\mathrm{M}+\mathrm{Na}^{6^{-}}\right.$(i.e., the amount of $\mathrm{Na}$ ions increases for lower charge states). Figure $3 \mathrm{~b}$ shows a spectrum of the same sample after addition of $25 \mathrm{mM}$ of piperidine and imidazole, and shows the reduction of $\mathrm{Na}$ adduction that allows an accurate mass measurement. Also note that the signal-to-noise ratio $(\mathrm{S} / \mathrm{N})$ improved as a

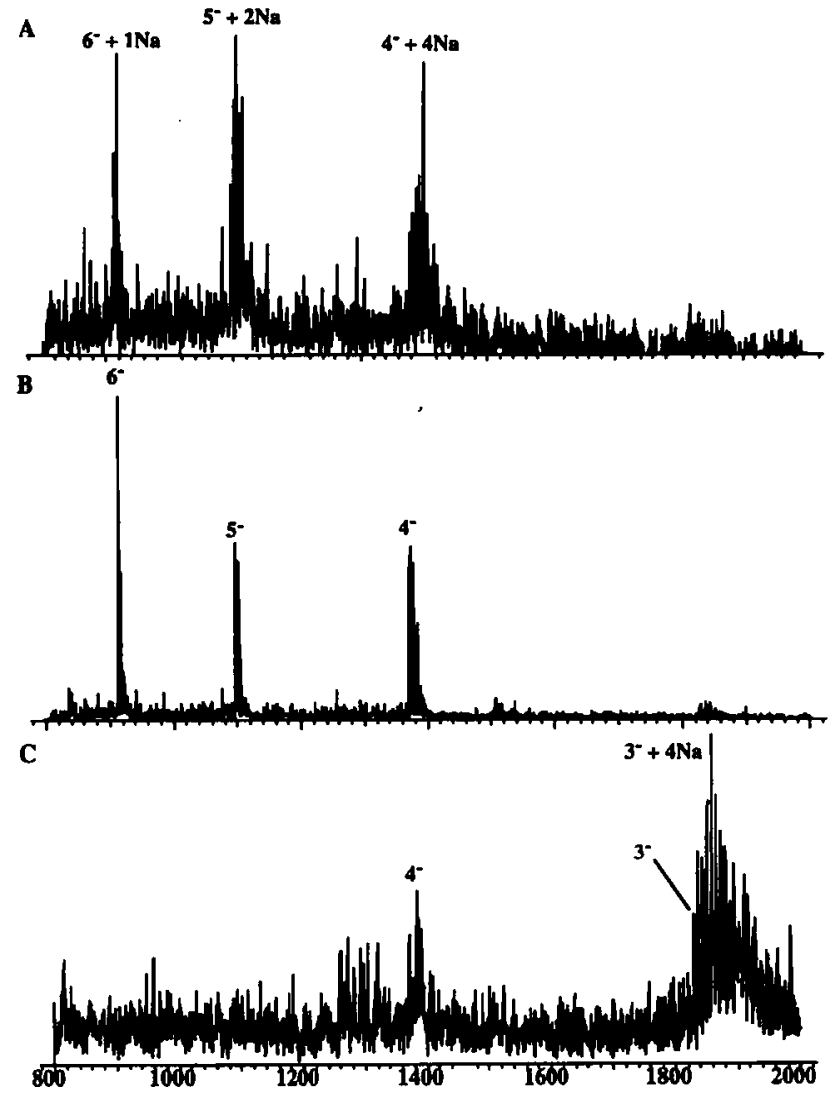

Figure 3. Electrospray ionization mass spectra of $d(\mathrm{pT})_{18}$ in (a) $80 \% \mathrm{ACN} / 10-\mathrm{mM} \mathrm{NaCl}$, (b) $80 \% \mathrm{ACN} / 10-\mathrm{mM} \mathrm{NaCl} / 25-\mathrm{mM}$ piperidine $/ 25-\mathrm{mM}$ imidazole, and (c) the same solution composition as (b) with 2.5-M acetic acid.

result of the distribution of ion current over fewer mass-to-charge ratio values. Interestingly, no chargestate shifting was observed with imidazole and piperidine when a high level of $\mathrm{NaCl}$ was present. Figure $3 c$ shows the ESI mass spectrum of the same sample shown in Figure $3 b$ after addition of HOAc (2.5 M). The co-addition of piperidine and imidazole still suppressed cation adduction in the presence of HOAc, and the HOAc still effectively reduced charge states. However, because the charge state was reduced, more $\mathrm{Na}$ adduction was observed. The poorer $\mathrm{S} / \mathrm{N}$ ratio is attributed to three phenomena: (1) Na adduction typically reduces the total ion signal due to dispersion of the ion current over several peaks in a single charge state; (2) poorer detection response at higher mass-tocharge ratio; (3) the addition of HOAc may shift the charge states outside the mass-to-charge ratio range investigated.

To verify if an interaction of piperidine and/or inidazole with oligonucleotides could be detected, the capillary temperature was reduced to $90^{\circ} \mathrm{C}$ to provide gentler desolvation conditions. Figure 4a shows the ESI mass spectrum of $T_{18}$ obtained from a solution that contained $80 \% \mathrm{ACN}$ and $25 \mathrm{mM}$ of piperidine and imidazole. Extensive adduction of piperidine was observed for all four charge states detected. Clearly, the charge states still have been shifted to higher mass-to- 
$\underline{A}$
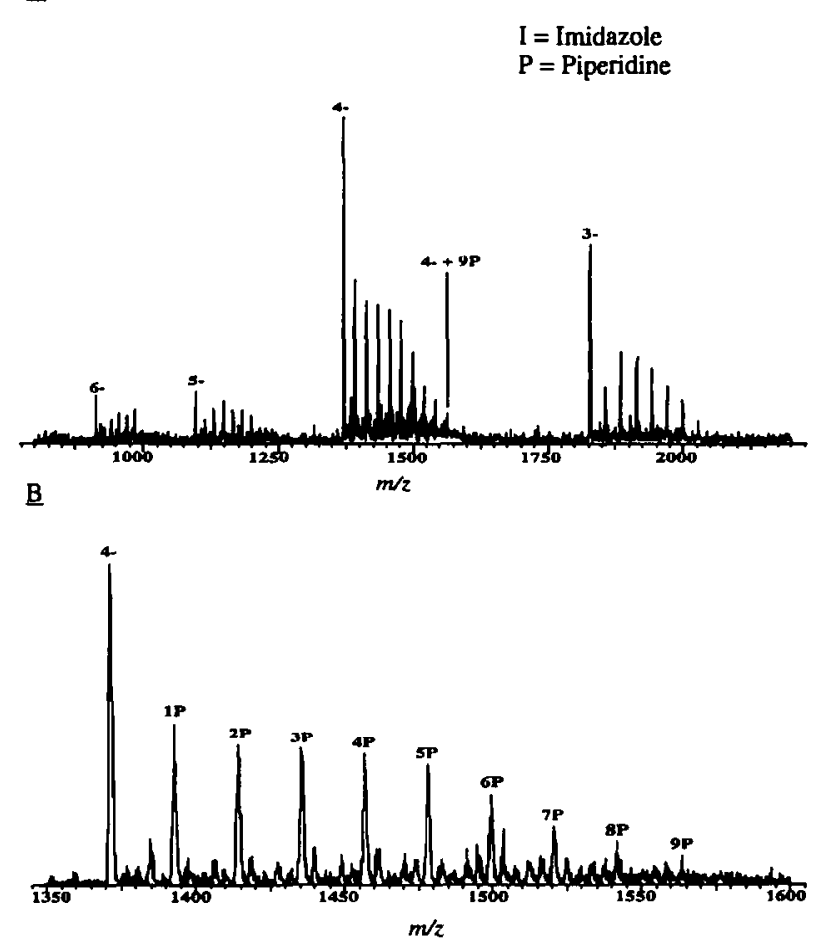

Figure 4. (a) Mass spectrum of $d\left(\mathrm{pT}_{18}\right.$ in $80 \% \mathrm{ACN} / 25-\mathrm{mM}$ piperidine at a relatively "cool" capillary temperature of $90^{\circ} \mathrm{C}$. (b) Expansion plot of the $4^{-}$charge state shown in (a).

charge ratio even at low desolvation temperatures. Figure $4 \mathrm{~b}$ shows an expanded mass scale of the $4^{-}$ charge state shown in Figure 4a. Interestingly, no adduction of imidazole was detected and this will be addressed vide infra.

\section{Oligonucleotide Mixture Analysis that Utilizes Solution Phase Chemistry}

Figure $5 \mathrm{a}$ shows the mass spectrum of a mixture that contained $\mathrm{d}(\mathrm{pA})_{6}, \mathrm{~d}(\mathrm{pT})_{18}$, and $\mathrm{d}(\mathrm{pC})_{12}$, each at a concentration of $\sim 60 \mu \mathrm{M}$ in $80 \% \mathrm{ACN}$. Although this mass spectrum is interpretable due to the fact that different sizes and few components are present, the addition of piperidine, imidazole, and HOAc resulted in a spectrum with lower spectral density as a result of the charge-state reduction as shown in Figure $5 \mathrm{~b}$. To evaluate how well the charge states of each oligonucleotide were reduced, the intensity of the lowest charge state observable of each component in the mass-to-charge ratio region studied $(800-2500)$ relative to the total intensity of each species was determined and the results are summarized in Figure 6a. The $T_{18}$ results are in direct accord with results shown in Figure $2 \mathrm{a}$ with a slight reversal of the FA and HOAc conditions, but are still within experimental error. We evaluated several of the previous solution conditions (Figure 2) and found that, in general, the reduction of charge states for each oligonucleotide as a function of solution composition was similar. It appears that the

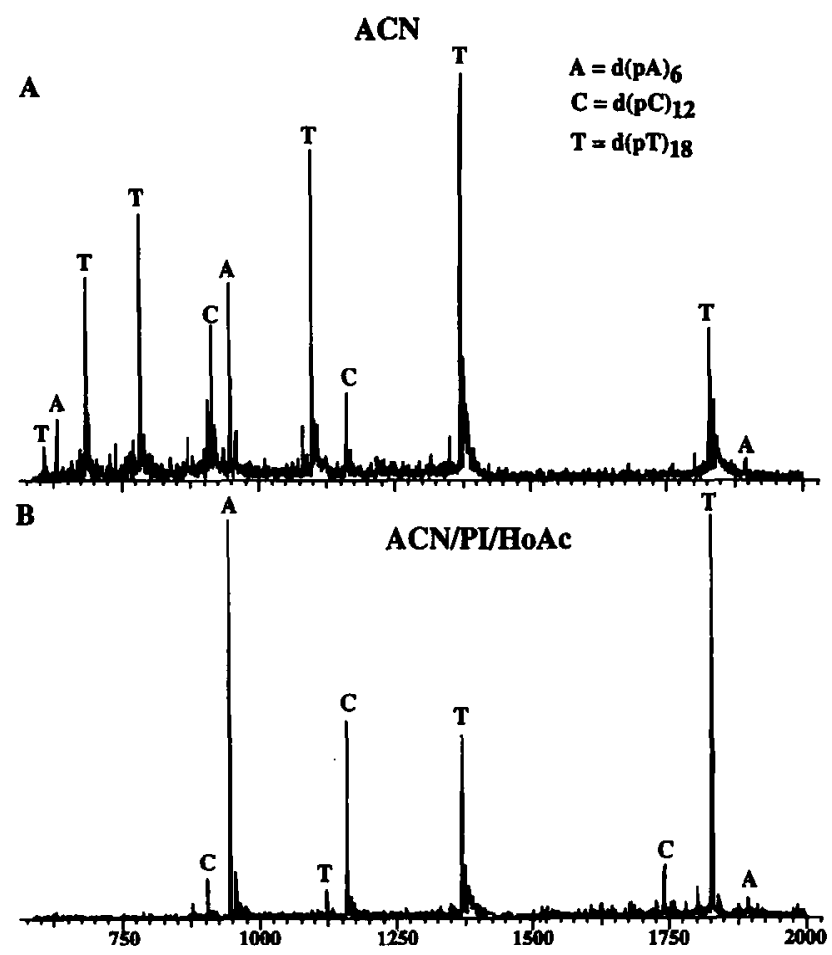

Figure 5. Electrospray ionization mass spectra of $d(p T)_{18}$, $\mathrm{d}(\mathrm{pA})_{6}$, and $\mathrm{d}(\mathrm{pC})_{12}$ in (a) $80 \% \mathrm{ACN}$ and (b) $80 \% \mathrm{ACN} / 25-\mathrm{mM}$ imidazole /25-mM piperidine/2.5-M acetic acid.

use of HOAc could reduce the charge-state distribution of $C_{12}$ only partially and had no effect on $A_{6}$, while at the same time almost all of the intensity of $T_{18}$ shifted into the $3^{-}$charge state. Also note that the absolute intensity of each oligonucleotide (i.e., intensity of all observed charge states) as a function of solution composition changed in similar fashion (data not shown).

The fraction of the total intensity of each oligonucleotide as a function of solution conditions is plotted in Figure 6b; the solution conditions are plotted with increasing efficiency of charge-state reduction (see Figure $6 a$ ). It is evident from Figure $6 \mathrm{~b}$ that there is a drop in the $T_{18}$ intensity $(\sim 25 \%)$ and an increase in both the $A_{6}$ and $C_{12}$ fractional ion intensity as the charge states are reduced. This drop in $T_{18}$ intensity relative to the two smaller oligonucleotides is attributed to the production of lower charge states of $T_{18}$ outside the mass-to-charge ratio range investigated.

To investigate this hypothesis as well as determine if it is a base- and/or size-related phenomenon, smaller bases were employed. Figure 7a shows the extent of charge-state reduction (intensity of the lowest charge state of $d N /$ total intensity of $d N$ ), where $d N$ represents each of the three deoxynucleotides investigated $\left(A_{8}, C_{8}\right.$, and $\left.T_{8}\right)$. The lowest charge state observed for all the oligonucleotides investigated was $2^{-}$. Figure $7 \mathrm{a}$ indicates that all three bases are reduced to the same extent as a function of solution composition. The fraction of the total intensity of each oligonucleotide as a function of solution conditions is plotted in Figure $7 \mathrm{~b}$; 


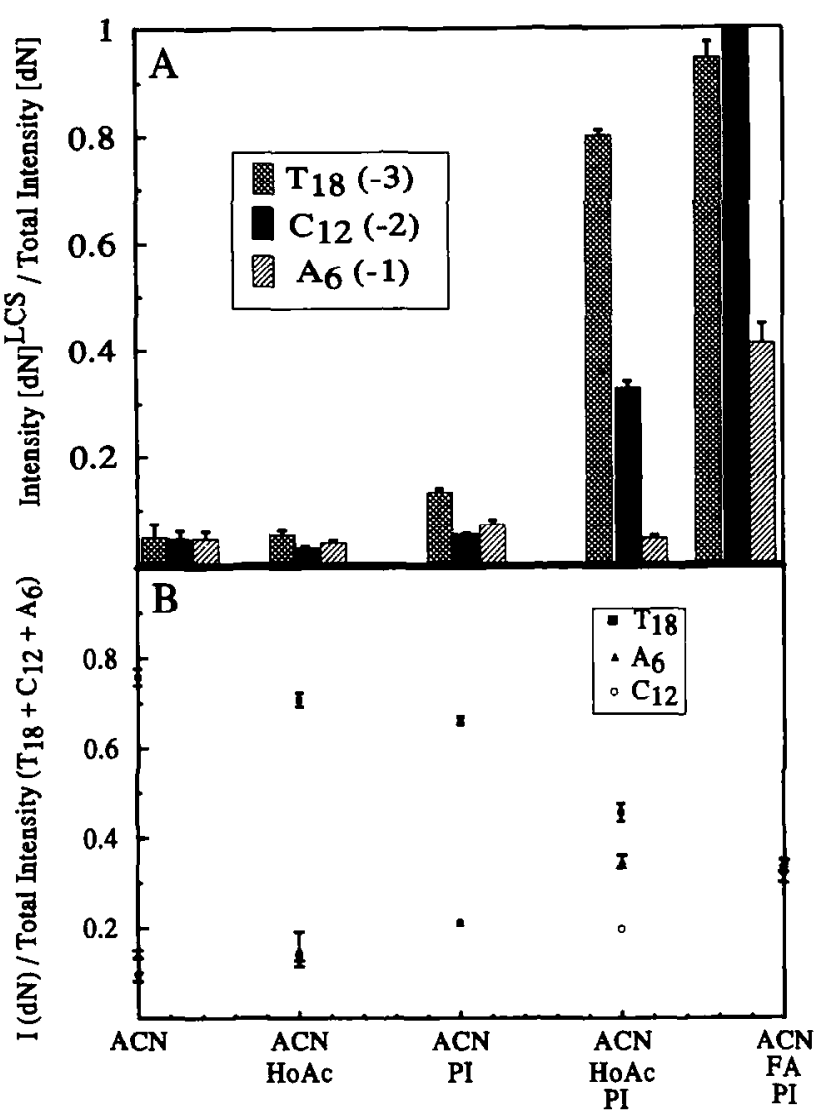

Figure 6. (a) Intensity of the lowest observable charge state of $\mathrm{d}(\mathrm{pT})_{18}, \mathrm{~d}(\mathrm{pA})_{6}$, and $\mathrm{d}(\mathrm{pC})_{12}\left(\mathrm{~d}[N]^{\mathrm{LCS}}\right)$ relative to the total intensity of each oligonucleotidc $(\mathrm{d}[N])$ as a function of different solution composition. (b) Intensity of d(pT) $18, \mathrm{~d}(\mathrm{pA})_{6}$, and $\mathrm{d}(\mathrm{pC})_{12}$ normalized to the total intensity of all three oligonucleotides as a function of solution composition.

the solution conditions are plotted with increasing efficiency of charge-state reduction (see Figure 7a). It is fortuitous that the fractional ion intensity is $\sim 0.3$ (i.e., the three oligonucleotides may not have the same sensitivity) and is attributed to the uncertainty in the amount of material provided by the manufacturer and losses that occur from sample handling. In these experiments where all charge states are observable, the fractional ion intensity remains constant as a function of the extent of charge-state reduction.

Figure 8a shows an ESI mass spectrum of a solution that contained $\mathrm{T}_{18}, \mathrm{C}_{12}, \mathrm{~A}_{6}, \mathrm{~A}_{8}, \mathrm{C}_{8}$, and $\mathrm{T}_{8}$ in a solution that contained $80 \% \mathrm{ACN}$. Figure $8 \mathrm{~b}$ shows a sample with the same oligonucleotide composition but with HOAc ( $2.5 \mathrm{M})$, piperidine $(25 \mathrm{mM})$, and imidazole $(25 \mathrm{mM})$ added. The most obvious difference between Figure $8 a$ and $b$ is the shift of charge states to higher mass-to-charge ratio. Thus, addition of two organic bases and an organic acid to a six-component mixture of oligonucleotides in an aprotic solvent reduces the spectral density (the number of peaks above $5 \%$ threshold value in the $m / z \quad 500-2000$ range by $\sim 40 \%$. In Figure 8a the peaks 1, 2, and 3 centered about $m / z 800$ and $1250 \mathrm{u}$ have extensive $\mathrm{Na}$ adduc-

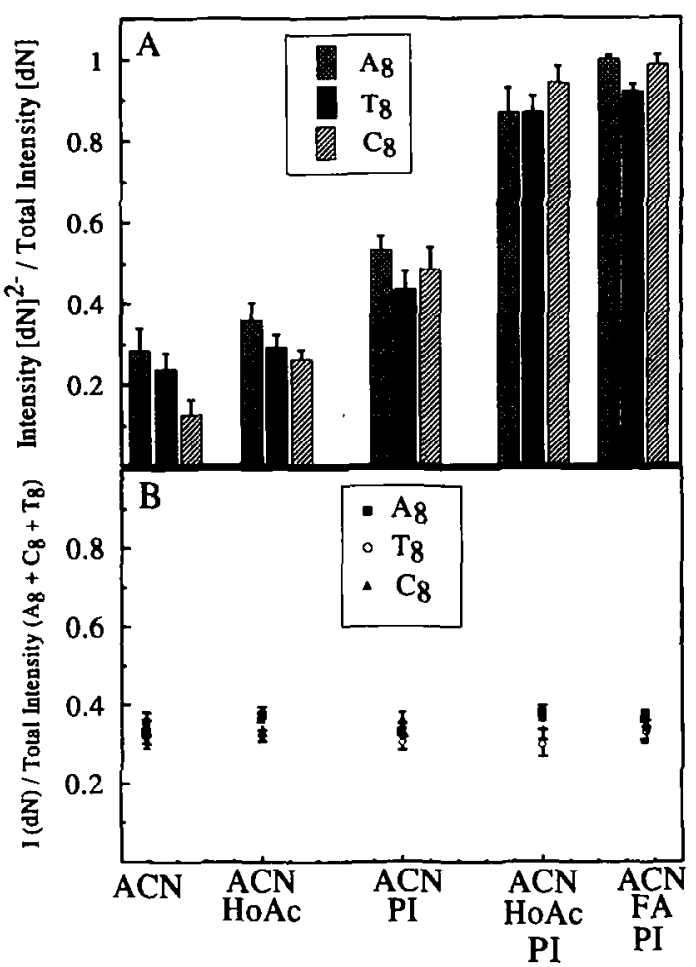

Figure 7. (a) Intensity of the lowest observable charge state of $\mathrm{d}(\mathrm{pT})_{8}, \mathrm{~d}(\mathrm{pA})_{8}$, and $\mathrm{d}(\mathrm{pC})_{8}\left(\mathrm{~d}[N]^{2-}\right)$ relative to the total intensity of each oligonucleotide $(\mathrm{d}[N])$ as a function of different solution composition. (b) Intensity of $\mathrm{d}(\mathrm{pT})_{8}, \mathrm{~d}(\mathrm{pA})_{8}$, and $\mathrm{d}(\mathrm{pC})_{8}$ normalized to the total intensity of all three oligonucleotides as a function of solution composition.

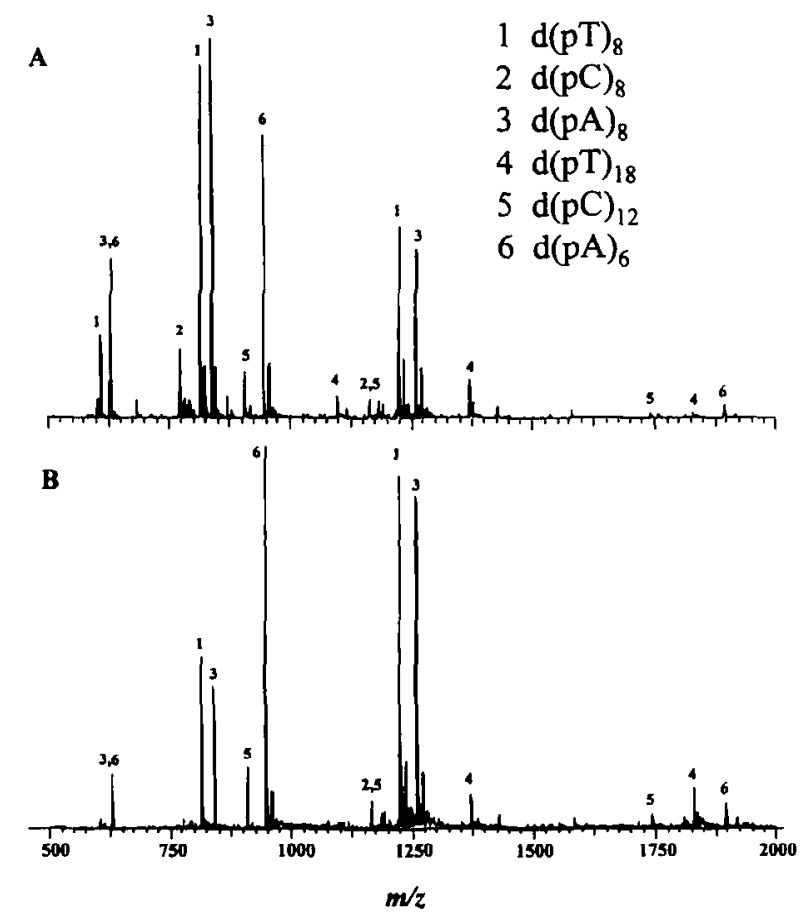

Figure 8. ESI mass spectrum of $d(p T)_{18}, d(p C)_{12}, d(p A)_{6}$, $\mathrm{d}(\mathrm{pA})_{8}, \mathrm{~d}(\mathrm{pT})_{8}$, and $\mathrm{d}(\mathrm{pC})_{6}$ obtained from a solution that contained (a) $80 \% \mathrm{ACN}$ and (b) $80 \% \mathrm{ACN} / 25-\mathrm{mM}$ piperidine/25$\mathrm{mM}$ imidazole/2.5-M HOAc. 
tion. The $2^{-}$charge state of the three 8-mers centered at $1250 \mathrm{u}$ have $\mathrm{Na}$ adduction of about $30 \%$ relative to the base peak $\left[(\mathrm{M}-2 \mathrm{H})^{2-}\right]$. However, in comparison, the 8-mer peaks centered around $m / z 800$ in Figure $8 b$ indicated no detectable amounts of $\mathrm{Na}$ adduction and the level of $\mathrm{Na}$ adduction of the $2^{-}$charge state has been reduced to about $10 \%$ relative to the unadducted species. Last, in this particular mixture, two components have overlapping charge states (i.e., the $4^{-}$charge state of $\mathrm{A}_{8}$ with the $3^{-}$charge state of $\mathrm{A}_{6}$ and the $3^{-}$ charge state of $C_{12}$ with the $2^{-}$charge state of $C_{8}$ ). Reduction of charge states (shifting to higher mass-tocharge ratio) will decrease the probability of such overlaps.

\section{Discussion}

The reduction of charge states of oligonucleotide negative ions by alteration of the solution composition facilitates the analysis of DNA mixtures. Production of fewer charge states compresses the intensity to fewer mass-to-charge ratio values and thus should provide better detection limits. Although it is difficult to determine at this point whether the charge states are reduced as a result of solution equilibria or gas-phase proton transfer, clearly charge states can be reduced by alteration of the solution composition. From the data presented in this paper, several explanations as to why the addition of acids and bases to a solution that contains an oligonucleotide has a "combined" effect should be addressed. The issue of cation suppression, charge-state reduction by using organic bases, and indirect and direct evidence that indicates the formation of lower charge states also will be addressed.

\section{Suppression of Cation Adduction to the Polyanionic Backbone of Oligonucleotides}

Two possible mechanisms for the reduction in $\mathrm{Na}$ adduction to DNA by using piperidine and imidazole are proposed. The suppression of $\mathrm{Na}$ adduction by using piperidine and imidazole is the competition of the piperidine and imidazole with the phosphodiesters for $\mathrm{Na}$ binding and competition of piperidine and imidazole with $\mathrm{Na}$ for phosphodiester binding. The first relationship depends on the affinity of piperidine and imidazole to $\mathrm{Na}$; the second depends on the affinity to the phosphodiester; however, both depend on basicity.

The polyanionic phosphodiester backbone of DNA is acidic $\left(p K_{a}<2\right)$. However, two resonance structures exist for the phosphate group. This duality delocalizes the electron distribution over the $\mathrm{P}-\mathrm{O}$ bonds almost equally in solution (which makes the formal charge on the ionizable oxygen misleading). Although this oxygen atom can attract sodium counterions in solution, more favorable interactions with strong "hard" bases can compete easily for the sodium ions in solution.
Piperidine is a strong Lewis base with the lone electron pair on the nitrogen able to form electrostatic interactions with cations in solution, in particular, sodium and potassium [20]. Sodium and potassium are considered to be "hard" acids due to the low polarizability of their electron clouds. Therefore, the suppression of cation adduction with oligonucleotides is attributed to the formation of strong electrostatic interactions between piperidine and $\mathrm{Na}^{+}$in solution. The amount of sodium chloride added in Figure 3 was $10 \mathrm{mM}$ and piperidine was present at $25 \mathrm{mM}$, so one might expect nearly complete suppression of cationization with $\mathrm{Na}$. However, each $\mathrm{Na}^{+}$in solution can interact with several piperidine molecules in solutions. This idea is supported by the observation that imidazole, a weak Lewis base, does not coordinate cations in solution as effectively as piperidine; this results from the fact that the imidazole is an aromatic nitrogenous base where the lone pair electrons of the nitrogen participate in the $\pi$ system of the five-member ring.

Inspection of Figure 3 also indicates that imidazole-piperidine does not reduce charge states in a solution that contains a substantial amount of salt. This result may indicate that both organic bases are "tied $\mathrm{up}^{\prime \prime}$ by the $\mathrm{Na}$ ions, which deactivates the imidazole-piperidine hydrogen bonding capabilities with the phosphate backbone due to the shift in equilibrium. However, the addition of acetic and formic acids to the oligonucleotide solutions that contain high levels of sodium still effectively reduces charge states.

Another mechanism with which $\mathrm{Na}$ adduction with oligonucleotides could be suppressed is the competition of piperidine and imidazole with $\mathrm{Na}$ for binding to the phosphate backbone of the DNA. In this mechanism, the piperidine and imidazole hydrogen bond to the phosphate backbone, thereby displacing $\mathrm{Na}$ ions. Upon entrance into the gas phase, a neutral loss of piperidine and imidazole occurs, which leaves the DNA molecule protonated. It is difficult to ascertain which mechanism dominates the removal of ubiquitous $\mathrm{Na}$ from the backbone, but both should be considered when developing new strategies for efficient $\mathrm{Na}$ removal from solutions that contain oligonucleotides.

\section{Determination of the Mechanistic Role of Imidazole and Piperidine in Reduction of the Charge States of Oligonucleotides}

Whereas the phosphate backbone is very acidic ( $\mathrm{p} K_{a}$ $<2$ ) and the $\mathrm{p} K_{a} \mathrm{~s}$ of HOAc and FA are 4.75 and 3.75, respectively, the phosphate backbone would not be expected to be protonated, especially in the presence of piperidine - a strong organic base. In fact, piperidine would be predicted to deprotonate the backbone, which would result in more extensive charging. However, there is a complete reversal of the relative basicities in the gas phase because the proton affinities (PA) of piperidine and imidazole are 226.4 [21] and 223.5 
$\mathrm{kcal} / \mathrm{mol}$ [22], respectively, and the PA of the phosphodiester functionality is estimated to be 315 $\mathrm{kcal} / \mathrm{mol}$ [23] due to the increased polarizability and intramolecular $\mathrm{H}$ bonding in DNA. Thus, when the oligonucleotide ion is transferred to the gas phase, the proton that originally resided on the organic base would "prefer" to reside on the phosphate backbone. For this to happen with high efficiency, the organic base likely will have to reside in close proximity to the phosphate backbone before desolvation. The formation of diamine adducts in solution followed by dissociation in the interface region was previously shown to lead to reduced charge states [11]. The interaction in solution was through hydrogen bonding, and because both nitrogenous bases (piperidine and imidazole) can hydrogen bond, essentially the same mechanism is proposed here. This proposed mechanism is illustrated in Figure 9, which shows the first three bases of a poly-C. The $5^{\prime}$ phosphate has imidazole hydrogen bonding to it, the second phosphate is charged, and the third phosphate has piperidine hydrogen bonding to it. Specifically, the piperidine and imidazole are hydrogen bonding to the phosphate backbone in solution and upon transfer to the gas phase, a neutral loss of either piperidine or imidazole occurs, which leaves the proton on the phosphate group (i.e., charge-state reduction). Thus, with reference to Figure 9, this portion of the poly- $\mathrm{C}$ would have only one of three possi-

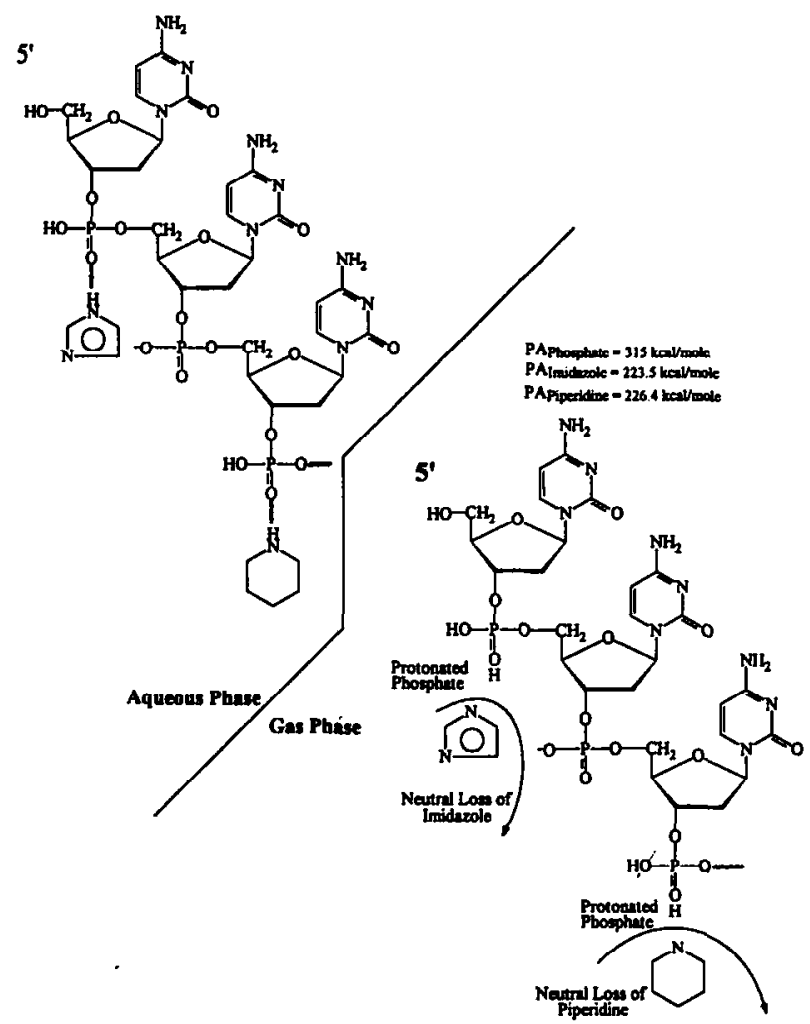

Figure 9. Proposed mechanism of the charge-state reduction of oligonucleotides negative ions by using piperidine and imidazole in the solution phase. The mechanism is illustrated by using the $5^{\prime}$ terminus of a poly-C. ble charges. Based on the previously stated proton affinities, this pathway is the most energetically favorable considering that it would be unlikely for opposite charges to separate in the gas phase (i.e., the protonated bases from the negatively charge phosphate group).

The role of the acetic acid in achieving more efficient charge state reduction may be in the protonation of the imidazole and piperidine; without the protonation of these bases in solution, the proposed mechanism in Figure 9 cannot occur. The $\mathrm{pH}$ of the electrosprayed solution that contains acetonitrile, piperidine, and imidazole is $\sim 11.5$, which is well above the $p K_{a}$ of imidazole; thus extensive adduction of imidazole is not expected because its hydrogen bonding capabilities are significantly reduced at higher $\mathrm{pH}$ values. If this is the role of the acid in the reduction of charge states in the presence of piperidine and imidazole, a low concentration $(10 \mu \mathrm{M})$ of a strong inorganic acid (e.g., $\mathrm{HCl}$ ) might prove to be a more useful approach. The role of the acid in the reduction of charge states is currently under investigation.

Additional support for this proposed mechanism (Figure 9) is shown in Figure 4 where extensive adduction of piperidine is observed. Although this adduction may be partially a result of aggregation during the ESI process [24], it is probable that some is due to the noncovalent interaction of piperidine with the phosphate backbone via hydrogen bonding. Inspection of Figure 4 indicates that for the $4^{-}$charge state, up to nine piperidine adducts are formed, which leaves only five protonated sites [based upon the 18 possible charges sites on $\left.\mathrm{d}(\mathrm{pT})_{18}\right]$. The average number of piperidine adducts likely is reduced in the interface region by dissociation, so it is impractical to be quantitative with regard to the extent of association. It seems unlikely that nine piperidines would be hydrogen bonded to the anionic backbone, which indicates that hydrogen bonding to the nucleotide bases may occur as well. Greig and Griffey [14] also postulated that imidazole interacted with the phosphate backbone and leaves as a neutral species. To substantiate this claim, they used $N$-methyl imidazole, which has a similar PA and $\mathrm{pK} \mathrm{K}_{a}$. They observed no charge-state reduction. However, imidazole has two nitrogens that can be involved in hydrogen bonding, whereas $\mathrm{N}$-methyl imidazole has only one nitrogen that can be involved in hydrogen bonding. Thus, $\mathrm{N}$-methyl imidazole can donate a single proton as well as the imidazole can.

\section{Are Lower Charge States Produced from Large Oligonucleotides?}

Indirect evidence exists that higher mass-to-charge ratio ions are produced, which alters only solution-phase compositions. Inspection of Figure $2 \mathrm{~b}$ shows that the absolute intensity for $T_{18}$ obtained from an $\mathrm{ACN} / \mathrm{HOAc}, \mathrm{ACN}, \mathrm{ACN} / \mathrm{PI}$, or HOAc solution is substantially higher than that obtained from a solution 
that contains ACN/PI/FA or ACN/PI/FA. Although the $\mathrm{FA} / \mathrm{ACN}$ has a low relative molecular ion intensity, this is explained by the FA that competes for the constant amount of ion current in the electrospray beam. However, solutions that contain ACN/PI with either FA or HOAc showed a marked drop in absolute intensity of molecular ions. This drop is attributed to the presence of lower charges states (i.e., $2^{-}$and $1^{-}$) that are produced but are not detected because they fall outside the mass-to-charge ratio range investigated. Although the intensity is low for all solutions that contain FA, solutions that contain HOAc without piperidine or imidazole were more intense than those that contain both organic bases.

Another investigation used oligonucleotides of different length and base composition to monitor the fraction ion intensity (see Figure 6). The intensity of the $d(p T)_{18}$ relative to the total intensity of all three oligonucleotides remained constant when only a small percentage of the lowest observable charge-state (3for $T_{18}$ ) was present. However, when a substantial amount of the $3^{-}$charge state was present, the fractional ion intensity of $T_{18}$ was reduced, which suggests that higher mass-to-charge ratio ions are produced. To further investigate this hypothesis, three small oligonucleotides were used and the same experiments were carried out (see Figure $7 \mathrm{~b}$ ). In this case, the fractional ion intensity remained constant (within experimental error), which further supports this suggestion.

Recently, direct evidence for the production of lower charge states of $\mathrm{d}(\mathrm{pT})_{18}$ was obtained on a Fourier transform ion cyclotron resonance mass spectrometer by using broad-band quadrupolar excitation over the mass-to-charge ratio range of 1500-3000 (Muddiman, D. C., unpublished results). This result clearly indicated the presence of the $2^{-}$charge state $(\sim 30 \%$ higher in intensity than the $3^{-}$charge state) when infused from a solution that contained acetonitrile, piperidine, and imidazole. This result is expected when we consider the "charge-state ladder" obtained for identical solution composition shown in Figure $1 \mathrm{~b}$.

\section{Protocol for Reduction of Charge States of Negative Oligonucleotide Ions}

From the results obtained, a sample preparation protocol to simplify the electrospray mass spectrum of a mixture of oligonucleotides can be presented. First, it is clear that the use of acetic acid provides the best signal intensities and that formic acid reduces analyte signal. Also, the latter has been reported to precipitate larger oligomers [13]. The use of acids stronger than acetic acid (such as trichloro and trifluro acetic acid) resulted in poor electrospray stability (data not shown). Thus, although acetic acid is weaker than formic acid and trichloro and trifluro acetic acid, acetic acid appears to be the best of those acids investigated thus far. Piperidine and imidazole are advantageous in conjunc- tion with acetic acid to aid in the reduction of charge states as well as to reduce $\mathrm{Na}$ and $\mathrm{K}$ adduction. The major role of piperidine (which is a strong Lewis base) is to bind "hard" cations in solution, whereas the major role of the imidazole is to reduce charge states (if protonated) with the added benefit of enhancement signal intensity. Although adduction of piperidine and imidazole was observed at low capillary temperatures, no adduction was observed at $160^{\circ} \mathrm{C}$ for the mass-tocharge ratio range studied. However, it remains to be determined if difficulties will arise for larger oligomers due to possible incomplete desolvation of piperidine and imidazole. Recently, it was reported that the use of piperidine and imidazole in a high organic phase for the ESI-MS analysis of a mixed base 49-mer drastically reduced cation adduction without observation of piperidine adducts [25]. Last, the sensitivity was enhanced greatly by using $80 \%$ acetonitrile and this should have no deleterious effects on larger oligomers.

\section{Conclusions}

The charge states of negatively charged oligonucleotide ions produced in an electrospray source could be reduced (i.e., shifted to higher mass-to-charge ratio) simply by alteration of the solution conditions of the sample to be infused. The absolute intensity of oligonucleotides could be maintained and a stable ESI current was achieved easily by using $\mathrm{ACN}$ in the presence of the charge-state reducing agent(s). The suppression of $\mathrm{Na}$ adduction by using the organic bases piperidine and imidazole was found to be reproducible and to be an effective way to remove ubiquitous cations from adducting to single-stranded DNA. Indirect evidence indicates the production of higher mass-to-charge ratio ions that subsequently will reduce the spectral density to facilitate the analysis of oligonucleotide mixtures.

\section{Acknowledgments}

We would like to thank Dr. Alan L. Rockwood and Dr. Joesph Manna (University of Utah) for helpful discussions and Dr. Amy C. Harms for instrumental assistance. This work was supported by the Human Genome Program, Office of Health and Environmental Research, the U.S. Department of Energy. Pacific Northwest Laboratory is operated by Battelle Memorial Institute for the U.S. Department of Energy, through contract DE-AC06-76RLO 1830 .

\section{References}

1. Fenn, J. B.; Mann, M.; Wang, C. K.; Wong, S. F.; Whitehouse, C. M. Mass Spectrom. Rev. 1990, 9, 37.

2. Smith, R. D.; Loo, J. A.; Edmonds, C. G.; Barinaga, C. J.; Udseth, H. R. Anal. Chem. 1990, 62, 882.

3. Smith, R. D.; Loo, J. A.; Ogorzalek Loo, R. R.; Busman, M.; Udseth, H. R. Mass Spectrom. Rev., 1991, 10, 359.

4. Yamashita, M.; Fenn, J. B. J. Phys. Chem. 1984, 88, 4451.

5. Karas, M.; Hillenkamp, F. Anal. Chem. 1988, 60, 2299. 
6. Tanaka, K.; Waki, H.; Ido, Y.; Akita, S.; Yoshida, T. Rapid Commun. Mass Spectrom. 1988, 2, 151.

7. Karas, M.; Bahr, U. Mass Spectrom. Rev. 1991, 10, 335.

8. Hillenkamp, F.; Karas, M.; Beavis, R. C.; Chait, B. T. Anal. Chem. 1991, 63, 1193A.

9. Chowdhury, S. K.; Katta, V.; Chait, B. T. Biochem. Biophys. Res. Commun. 1990, 167, 686.

10. Perkins, J. R.; Smith, B.; Gallagher, R. T.; Jones, D. S.; Davis, S. C.; Hoffman, A. D.; Tomer, K. B. J. Am. Soc. Mass Spectrom. 1993, 4, 670.

11. Cheng, X.; Gale, D. C.; Udseth, H. R.; Smith, R. D. Anal. Chem. 1995, 67, 586.

12. Sanger, F.; Nicklen, S.; Coulson, A. R. Proc. Natl. Acad. Sci. U.S. A. 1977, 74, 5463.

13. Stults, J. T.; Marsters, J. C. Rapid Commun. Mass Spectrom. 1991, 5, 359.

14. Greig, M.; Griffey, R. H. Rapid Commun. Mass Spectrom. 1995, $9,97$.

15. Potier, N.; Van Dorsselaer, A.; Cordier, Y.; Roch, O.; Bischoff, R. Nucleic Acids Res. 1994, 22, 3895.

16. Grotjahn, L.; Blocker, H.; Frank, R. Biomed. Mass Spectrom. $1985,12,514$.
17. Limbach, P. A.; Crain, P. F.; McCloskey, J. A. J. Am. Soc. Mass Spectrom. 1995, 6, 27.

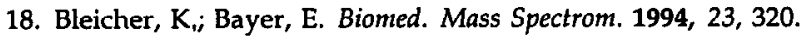

19. Gale, D. C; Smith, R. D. Rapid Commun. Mass Spectrom. 1993, 7, 1017.

20. Huheey, J. E.; Keiter, E. A.; Keiter, R. L. Inorganic Chemistry: Principles of Structure and Reactivity; Harper Collins College Publishers: New York, 1993.

21. Lias, S. G.; Liebman, J. F.; Levin, R. D. J. Chem. Phys. Ref. Data 1984, 12, 695.

22. Cataian, J.; Claramunt, R. M.; ,Elguero, J.; Laynez, J.; Menendez, M.; Anvia, F.; Quian, J. H.; Taagepera, M.; Taft, R. W. J. Am. Chem. Soc. 1988, 110, 4105.

23. Lum, R. C.; Grawbowski, J. J. J. Am. Chem. Soc. 1992, 114, 8619.

24. Smith, R. D.; Light-Wahl, K. J.; Winger, B. E.; Loo, J. A. Org. Mass. Spectrom. 1992, 27, 811.

25. Wunschel, D. S.; Fox, K. F.; Fox, A.; Bruce, J. E.; Muddiman, D. C.; Smith, R. D. Rapid Commun. Mass Spectrom., 1996, 10, 29. 